\title{
Abilities of preschoolers: comparing different tools
}

\author{
Matteo Chiappedi ${ }^{1 *}$, Erika Maffioletti ${ }^{2}$, Fausta Piazza ${ }^{2}$, Nicole D'Adda ${ }^{2}$, Marta Tamburini ${ }^{2}$ and Umberto Balottin ${ }^{2}$
}

\begin{abstract}
Background: There is a strong need for studies evaluating tests in terms both of psychometric properties (i.e. their efficacy or ability to be helpful in reaching a diagnosis) and of their cost-effectiveness (i.e. their efficiency). These data are essential for planning a correct evaluation to identify children's needs (both educational and abilitative).

Methods: We evaluated 58 children attending for the first time the last year of the Scuola dell'Infanzia. Parental view was obtained with Child Behaviour Check-List and Conners' Rating Scales - Revised, and family socioeconomic status was evaluated using Hollingshead's Four Factor Index; teacher compiled the IPDA questionnaire; children were administered Raven's Progressive Matrices, Modified Bell Cancellation Test, BVN 5-11 (a neuropsychological battery).

Results: A correlational analysis was conducted using Spearman's Rho (since variables were not normally distributed). These asymptomatic children show a good global cognitive functioning, but also a deficit of attention and of Executive Functions. Some of the tests used seem more cost-effective than others and there are some redundancies in information obtained.

Conclusions: Our data show that there are significant correlations between different neuropsychological and behavioural measures. It is therefore possible to rationalize diagnostic protocols without a significant information reduction. A deeper analysis will require a preliminary definition of the psychometric properties of used tools.
\end{abstract}

\section{Background}

Different neuropsychological functions have been shown in pre-schoolers to be more closely correlated to successive school achievement [1]: the integration of several cognitive and perceptual-motor skills is required since the beginning of primary school [2]. English-based literature has focused on letter recognition, spelling ability, phonemic awareness for reading and writing [1,3] and number recognition, quantity processing and counting for mathematical skills $[4,5]$. Given that Italian, unlike English, has an almost fully transparent orthography, it is perhaps understandable that studies conducted in Italy have shown a preminent role of metaphonological skills [6]. It has been written that these abilities represent a crystallized knowledge, deriving from experiences conducted at home, at the nursery or in other social contests; the role of the so called "g factor" (fluid intelligence, independent from experience) has been stressed

\footnotetext{
* Correspondence: mchiappedi@dongnocchi.it

'Don Carlo Gnocchi ONLUS Foundation, Milan, Italy

Full list of author information is available at the end of the article
}

especially for higher level and more complex cognitive activities [7].

Executive Functions (EF) have been implied both in relational development in childhood and learning; they can be defined as cognitive processes implied in behavioural regulation and include cognitive flexibility, impulse control, working memory, goal-directed planning and regulation of activity [8]. The role of EFs has been demonstrated for both reading/writing and mathematical skills $[9,10]$,

Among EFs, a special role is probably played by attention, in its different forms and components $[11,12]$.

Learning disabilities are an important risk factor for academic dropout and can influence the social and emotional wellbeing of the child [13]. A learning disability can be cause and/or consequence of an emotional problem, which in turn can compromise academic and relational results [14]. A timely diagnosis of any deficit in skills connected with the possibility to learn is therefore important in order to plan an effective strategy to reduce these deficits and to maximize learning possibilities.

\section{Biomed Central}


In common neuropsychiatric practice, information is collected from different sources (children themselves, parents, teachers and so on) with different tools (including tests, questionnaires or clinical interviews). In slump time as now, when high costs are a problem and are increasingly reported for Health Systems of Western Societies, we need to be worried about improving not only the efficacy of our tools, but also their efficiency. It would be therefore useful to have data not only about psychometric properties of commonly used tests, but also about cost-effective strategies to use them.

\section{Methods}

Fifty-eight Italian children (25 females, 33 males) attending for the first time the last year of the pre-shool in the "Terzo Circolo" of Pavia were enrolled in this study; their age was between 5 and 6 years. We excluded children with signs of neurological or psychiatric disorders and/or born pre-term. All parents, acting as legal guardians, signed an informed consent and all data were collected and analyzed following the Helsinki declaration.

Children were evaluated in order to obtain information from three main sources, namely parents, teachers and children themselves. To this aim, we used:

1) for parents: Child Behavior CheckList, a questionnaire by Achenbach and co-workers which explores many different domains of functioning [15]; Conners' Rating Scales - Revised, a questionnaire meant to explore perceived behaviours connected with attention deficit and/or hyperactivity [16]; Hollingshead's Four Factor Index, a rather simple tool quantifying the socio-economic status of the family [17];

2) for teachers: IPDA questionnaire, by which the teacher is supposed to express a quantified evaluation of the child's functioning [18];

3) for children: Raven's Progressive Matrices (coloured form), to study learning independent and culture-free intelligence [19]; Modified Bell Cancellation Test, to evaluate attentive skills [20]; BVN 5-11, a battery of neuropsychological tests developed for children aged from 5 to 11 years old [21]. This part of the evaluation was administered during normal school time, inside the school, in a room commonly used for "attention requiring activities".

Descriptive statistics for all variables tested are depicted in Table 1.

We also tried to quantify the cost of the tools, taking into account the cost of the material used but also the amount of time needed to present the test.

We analyzed obtained data with MedCalc (TM) and SPSS-PC (TM) version 15 in order to compare these
Table 1 Descriptive statistics for tested variables

\begin{tabular}{|c|c|c|}
\hline Measure & Applied to & Range \\
\hline Raven's Progressive Matrices & 58 & $6-32$ \\
\hline \multicolumn{3}{|l|}{ Modified Bell Cancellation Test: } \\
\hline Rapidity & 58 & $6-51$ \\
\hline Accuracy & 58 & $30-129$ \\
\hline \multicolumn{3}{|l|}{ BVN 5-11 } \\
\hline Auditory discrimination & 58 & $60.4-119.7$ \\
\hline Non-words repetition & 55 & $87.9-122.2$ \\
\hline Phonemic analysis & 46 & $76.1-131.1$ \\
\hline Phonemic fusion & 31 & $90.0-177.3$ \\
\hline Naming & 58 & $61.9-140.6$ \\
\hline Syntactic comprehension & 57 & $0-136.6$ \\
\hline Digit span & 58 & $86.3-144.1$ \\
\hline Corsi Test & 58 & $68.1-132.1$ \\
\hline Word pairs learning & 53 & $80.9-174.6$ \\
\hline Word memory & 56 & $74.7-152.7$ \\
\hline Short term memory & 58 & $59.8-143.5$ \\
\hline Long term memory & 58 & $60.4-128.9$ \\
\hline Praxic verbal skills & 56 & $79.5-124.5$ \\
\hline Praxic imitative skills & 56 & $68.3-123.8$ \\
\hline Tower of London & 57 & $68.8-144.1$ \\
\hline Phonemic fluency & 54 & $86.9-147.5$ \\
\hline Categorical fluency & 58 & $64.2-130.0$ \\
\hline Visual discrimination & 57 & $75.0-117.2$ \\
\hline Visual quantity judgement & 57 & $43.1-128.1$ \\
\hline Auditory quantity judgement & 58 & $58.9-135.3$ \\
\hline Auditory attention & 56 & $34.4-141.5$ \\
\hline Visual attention & 55 & $77.8-139.0$ \\
\hline Counting & 58 & $40.5-98.0$ \\
\hline$\overline{\mathrm{CBCL}}$ & 56 & \\
\hline$\overline{C R S-R}$ & 55 & \\
\hline$\overline{I P D A}$ & 57 & $88-172$ \\
\hline Hollingshead's Four Factor Index & 54 & $12-63.5$ \\
\hline
\end{tabular}

Measures derived from BVN 5-11 have a range expressed in terms of Standard Score. CBCL and CRS-R do not have a reported range because many indexes can be calculated (see also Table 3). IPDA has a range expressed for the complete score. Hollingshead's Four Factor Index has a range expressed in terms of child's score for Socio-Economic Status.

tests and to identify a rational evaluation strategy to be used in asymptomatic children. Given that variables were ordinal but not normally distributed (as resulted from Kolgomorov-Smirnoff test) we used Spearman's Rho to evaluate correlations significance.

\section{Results}

\section{Subjects studied}

Descriptive statistics concerning our patients are depicted in Table 1. 
Socio-economic status, described following Hollingshead's recommendations [17], seems to mimic the distribution of the general population.

Most children have a good or excellent cognitive functioning (96.4\% scores higher than $50^{\text {th }}$ percentile in Raven's Progressive Matrices). Parents do not seem to identify any particular problem in their children, since mean values in both CBCL and Conners' Rating Scales - Revised are near 50 (i.e. normal, since these tests results are given as $\mathrm{T}$ scores); no child fell into the pathological range for any group of symptoms. Taken together, these data seem particularly good even considering that children with known psychiatric and/ or neurologic disorders were excluded from our sample.

On the other hand, teachers describe children in a more distributed way, with significant peaks in the highest functioning class $(50 \%$ of children obtained a score higher than $75^{\text {th }}$ percentile) and in the "frail but not poor group" ( $38 \%$ fell between the $10^{\text {th }}$ and the $25^{\text {th }}$ percentile).

As to BVN 5-11, most of the children performances were in the normal range but some tests of the battery were frequently refused; this is particularly true for Phonemic Fusion, a meta-phonological test which was accepted by only 31 children (53.4\%).

Most children showed a poor attentive performance at the Modified Bell Cancellation Test, both in terms of Rapidity (i.e. fixation attention; $73 \%$ of children fell below the $25^{\text {th }}$ percentile) and of Accuracy (i.e. sustained attention; $60 \%$ of children fell below the $25^{\text {th }}$ percentile).

\section{Correlation between scores}

Statistically significant correlations are given in Table 2 . It must be noted that a large number of comparisons

Table 2 Significant correlation between tests

\begin{tabular}{llll}
\hline Test & Correlates with & Significance & Correlation \\
\hline $\begin{array}{l}\text { Raven's } \\
\text { Progressive }\end{array}$ & Auditory discrimination & $P<0.001$ & -0.492 \\
\hline & & & \\
\hline & Phonemic analysis & $P=0.002$ & -0.458 \\
\hline & Corsi test & $P=0.007$ & -0.357 \\
\hline & Word pairs learning & $P=0.005$ & -0.386 \\
\hline & Auditory attention & $P=0.01$ & -0.347 \\
\hline & Tower of London & $P<0.001$ & -0.475 \\
\hline Visual discrimination & $P=0.005$ & -0.376 \\
\hline Cancellation Test: & & & \\
\hline Rapidity & Corsi test & $P=0.047$ & -0.262 \\
\hline & Short term memory & $P=0.001$ & -0.432 \\
\hline & Long term memory & $P=0.048$ & -0.260 \\
\hline
\end{tabular}

Table 2 Significant correlation between tests (Continued)

\begin{tabular}{|c|c|c|c|}
\hline & Auditory attention & $P=0.026$ & -0.297 \\
\hline & Tower of London & $P=0.01$ & -0.339 \\
\hline & Categorical fluency & $P=0.003$ & -0.388 \\
\hline \multirow[t]{10}{*}{ Correctness } & Auditory discrimination & $P=0.01$ & -0.336 \\
\hline & Corsi test & $P=0.022$ & -0.299 \\
\hline & Short term memory & $P<0.001$ & -0.454 \\
\hline & Long term memory & $P=0.014$ & -0.322 \\
\hline & Auditory attention & $P=0.021$ & -0.307 \\
\hline & Tower of London & $P=0.013$ & -0.326 \\
\hline & Categorical fluency & $P=0.008$ & -0.346 \\
\hline & Phonemic fluency & $P=0.001$ & -0.424 \\
\hline & Visual discrimination & $P=0.037$ & -0.277 \\
\hline & Social problems (CBCL) & $P=0.028$ & +0.297 \\
\hline \multirow{13}{*}{$\begin{array}{l}\text { Hollingshead's } \\
\text { Four Factor Index }\end{array}$} & Auditory discrimination & $P<0.001$ & -0.518 \\
\hline & Phonemic analysis & $P<0.001$ & -0.571 \\
\hline & Phonemic fusion & $P=0.02$ & -0.414 \\
\hline & Digit span & $P=0.003$ & -0.294 \\
\hline & Word pairs learning & $P=0.004$ & -0.396 \\
\hline & Visual attention & $P=0.023$ & -0.318 \\
\hline & Tower of London & $P=0.001$ & -0.432 \\
\hline & Phonemic fluency & $P<0.001$ & -0.673 \\
\hline & Categorical fluency & $P=0.004$ & -0.385 \\
\hline & Visual discrimination & $P=0.005$ & -0.381 \\
\hline & $\begin{array}{l}\text { Attention deficit's DSM } \\
\text { IV symptoms (CRS-R) }\end{array}$ & $P=0.04$ & +0.182 \\
\hline & $\begin{array}{l}\text { Raven's Progressive } \\
\text { Matrices }\end{array}$ & $P=0.0013$ & +0.340 \\
\hline & IPDA & $P<0.001$ & +0.511 \\
\hline \multirow[t]{10}{*}{ IPDA } & Auditory discrimination & $P=0.002$ & -0.410 \\
\hline & Phonemic analysis & $P=0.03$ & -0.325 \\
\hline & Word pairs learning & $P=0.012$ & -0.347 \\
\hline & Long term memory & $P=0.039$ & -0.275 \\
\hline & Phonemic fluency & $P=0.002$ & -0.403 \\
\hline & $\begin{array}{l}\text { Externalizing problems } \\
\text { (CBCL) }\end{array}$ & $P=0.045$ & +0.272 \\
\hline & Total problems (CBCL) & $P=0.034$ & +0.286 \\
\hline & Perfectionism (CBCL) & $P=0.017$ & +0.324 \\
\hline & $\begin{array}{l}\text { Psycho-somatic } \\
\text { problems (CBCL) }\end{array}$ & $P=0.012$ & -0.341 \\
\hline & SES & $P<0.001$ & +0.511 \\
\hline
\end{tabular}

were performed; although a stepwise approach was used, we cannot definitely rule out the existence of Type I errors (since this technique is known to be less conservative than, for instance, a Bonferroni correction).

As to Raven's Progressive Matrices, all correlations are positive (i.e. better results in other tests tends to predict a better result in Raven's Progressive Matrices). 
The same applies for the Modified Bell Cancellation Test; in this case, however, there are differences between Rapidity and Accuracy in terms of correlated tests.

Hollingshead's Four Factor Index correlates with many tests and subtests, again with a positive trend (i.e. a higher Socio-Economic Status predicts better results but also a higher level of parental perception of child's problems, particularly in terms of attention deficit).

The IPDA correlates with many other tests and subtests, among which the positive correlation with the Socio-Economic Status and the negative one with externalizing and general problems signalled by parents (CBCL scores) are particularly noteworthy (i.e. more problems correlate with a teacher's perception of reduced child's skills).

CBCL's and CRS-R's correlations are reported in Table 3. These two questionnaires correlate to each other in a positive way (i.e. children with higher reported problems in one questionnaire tend to have higher reported problems in the other questionnaire).

\section{Cost evaluation of different tools}

Table 4 presents the economic evaluation of all tests used in this study.

We present the cost of the basic kit; the cost of materials for a single administration (approximated to the whole euro), described as one hundredth of the cost of the basic kit plus the cost of any consumable material necessary; the time needed for a single administration (given in minutes and derived from the manual of the test but also from our experience with children in this study); the cost of the operator (administration and scoring), assuming a standardized full cost of 30 Euro per hour; the full cost of a single administration (resulting from the cost of the materials and the cost of the operator).

\section{Discussion}

Learning can be defined as a complex process, involving motivation, emotions, memory and other cognitive processes that are necessary to acquire meaningful information useful in reaching one or more specific goals. The assessment of learning possitibilities in a child should therefore include many different aspects, ranging from an evaluation of motivation and emotional balance to a wide range of cognitive skills [22].

Our study offers data concerning the correlations existing between different evaluation tools and analyzes the economical aspects of their use.

To start with, it should be stressed that the existence of a statistically significant correlation does not imply a cause - effect connection. In our study, correlations between some measures used were found.
Moreover, for most of the tests used (as well as for most tests used in child neuropsychiatry in general) psychometric properties are poorly defined; this constitutes a major limitation for this study but also, more importantly, a relevant problem for everyday clinical practice.

Teachers description of the child, quantified by the IPDA questionnaire, is correlated to child's ability to use language and to manipulate its parts (so called "metaphonological skills"). This was probably to be expected, given that teachers are supposed to be interested in the cognitive functioning and to exploit cognitive and linguistic skills to obtain learning. On the other hand, the reason why their views correlate with the Socio-Economic Status of the family and with parental perception of behavioural problems (especially externalizing problems) is not self-evident. Children from poorer families and/or whose parents report more externalizing problems (i.e. a tendency towards provocative or disruptive or hyperactive behaviours) tend to be seen as "less able" from their teachers, even when they have adequate neuropsychological skills; the interaction between these factors is not completely clear from our data.

One could speculate that externalizing behaviours could be "disturbing" for the learning process, but also for the teacher herself. This view, however, does not explain the SES factor.

Raven's Progressive Matrices are supposed to allow the investigation of intelligence in a culture-free and learning independent way. It is interesting to note that the score obtained by the child is significantly correlated to his attentive and visual processing skills: this could have been predicted from an analysis of the proposed test, which is based on the ability to perform a visual scanning of the matrices and to concentrate on the task. The correlation with Executive Functions and therefore with the ability to face complex and new situations in a successful way has been reported for different intelligence tests [23]. It is worth noting, as to this point, that Phonemic Analysis and Phonemic Fusion, which are considered subtests exploring the metaphonological correlates of Executive Functions, were performed by 46 (79.3\%) and 31 (53.5\%) of children only: this may imply that these children, although tested in a well-known setting and with a known adult to assist them, were unable even just to try to answer to this unfamiliar request.

Socio-Economic Status is known to influence cognitive development and a variety of cognitive abilities [24] and our data seem to confirm previous and widely reported findings. It should be noted, however, that most published reports have been obtained in children from English-speaking countries. Our data, confirming previous findings, stand with the hypothesis that considers SES as 
Table 3 Significant correlations between CBCL and CRS-R

\begin{tabular}{|c|c|c|c|}
\hline CBCL score & Correlates with CRS score & Correlation & Significance \\
\hline \multirow[t]{13}{*}{ Total } & Oppositivity & 0.639 & $P<0.001$ \\
\hline & Cognitive problems & 0.510 & $P<0.001$ \\
\hline & Hyperactivity & 0.543 & $P<0.001$ \\
\hline & Anxiety/Shyness & 0.647 & $P<0.001$ \\
\hline & Perfectionism & 0.366 & $P=0.006$ \\
\hline & Psychosomatic problems & 0.274 & $P=0.043$ \\
\hline & ADHD symptoms & 0.578 & $P<0.001$ \\
\hline & Fidgety/Impulsivity & 0.667 & $P<0.001$ \\
\hline & Emotional instability & 0.414 & $P=0.002$ \\
\hline & Clinical Global Impression & 0.662 & $P<0.001$ \\
\hline & Attention deficit (DSM IV) & 0.626 & $P<0.001$ \\
\hline & Hyperactivity (DSM IV) & 0.524 & $P<0.001$ \\
\hline & ADHD symptoms in DSM IV & 0.629 & $P<0.001$ \\
\hline \multirow[t]{13}{*}{ Internalizing problems } & Oppositivity & 0.585 & $P<0.001$ \\
\hline & Cognitive problems & 0.355 & $P=0.008$ \\
\hline & Hyperactivity & 0.447 & $P=0.001$ \\
\hline & Anxiety/Shyness & 0.569 & $P<0.001$ \\
\hline & Perfectionism & 0.312 & $P=0.02$ \\
\hline & Psychosomatic problems & 0.390 & $P=0.003$ \\
\hline & ADHD symptoms & 0.390 & $P=0.003$ \\
\hline & Fidgety/Impulsivity & 0.445 & $P=0.001$ \\
\hline & Emotional instability & 0.402 & $P=0.002$ \\
\hline & Clinical Global Impression & 0.461 & $P<0.001$ \\
\hline & Attention deficit (DSM IV) & 0.432 & $P=0.001$ \\
\hline & Hyperactivity (DSM IV) & 0.384 & $P=0.004$ \\
\hline & ADHD symptoms in DSM IV & 0.404 & $P=0.002$ \\
\hline \multirow[t]{12}{*}{ Externalizing problems } & Oppositivity & 0.567 & $P<0.001$ \\
\hline & Cognitive problems & 0.472 & $P<0.001$ \\
\hline & Hyperactivity & 0.505 & $P<0.001$ \\
\hline & Anxiety/Shyness & 0.433 & $P=0.001$ \\
\hline & Perfectionism & 0.407 & $P=0.002$ \\
\hline & ADHD symptoms & 0.624 & $P<0.001$ \\
\hline & Fidgety/Impulsivity & 0.718 & $P<0.001$ \\
\hline & Emotional instability & 0.354 & $P=0.008$ \\
\hline & Clinical Global Impression & 0.700 & $P<0.001$ \\
\hline & Attention deficit (DSM IV) & 0.560 & $P<0.001$ \\
\hline & Hyperactivity (DSM IV) & 0.515 & $P<0.001$ \\
\hline & ADHD symptoms in DSM IV & 0.608 & $P<0.001$ \\
\hline
\end{tabular}

a relevant factor in determining one's cognitive development.

CBCL and Conners' Rating Scales - Revised seem to be rather similar in providing information about parental view. This finding is not new, as previous research with children with ADHD has shown that the inclusion of multiple parent questionnaires does not lead to incremental validity [25]. Our study adds to the demonstration that these questionnaires are widely correlated also in a non-clinical population.

It must be stressed that CBCL and CRS-R profile depend on the real functioning of the child but also on the interpretation of this functioning given by parents; this might explain why parents seemed not to consider their children affected by an attention deficit although most of them performed poorly in an attention test 
Table 4 Cost of tests used

\begin{tabular}{llllll}
\hline TEST & Base kit cost & Materials cost & Time needed & Operator cost & Full cost \\
\hline BVN 5-11 & 169 & 5 & 180 & 90 & 95 \\
\hline Raven's Progressive Matrices & 275 & 3 & 40 & 20 & 23 \\
\hline Modified Bell Cancellation Test & 0 & 1 & 20 & 10 & 11 \\
\hline CBCL & 248 & 9 & 20 & 10 & 19 \\
\hline Conners' Rating Scales - Revised & 263 & 11 & 15 & 7,5 & 18,5 \\
\hline IPDA & 29 & 3 & 20 & 10 & 13 \\
\hline Hollingshead's Four Factor Index & 0 & 0 & 10 & 5 & 5 \\
\hline
\end{tabular}

(Modified Bell Cancellation Test). This attention test has published normative data for this age class [20]: this suggests that most children show a real difficulty in the attentive field and is consistent with the difficulties we found for Visual and Auditory Attention as measured in the BVN 5-11.

It is also worth noting that the perception of the child problems tended to increase in families with a higher Socio-Economic Status, showing that the score is probably influenced by parent's view of how a child should behave.

BVN 5-11 is the only tool in our research protocol which allows to reach a wide functional profile of the child, which can be predicted only partially through the other tools used. This can be useful to identify specific deficits and to plan an adequate intervention, both in terms of an adequate kindergarten activity and/or of an abilitative treatment.

Taking costs into account (see Table 4), Raven Progressive Matrices seem to be not only valuable (as they quantify cognitive functioning) but also cost-effective.

CBCL and Conners' Rating Scales - Revised seem to overlap in terms of diagnostic utility and cost: in order to reduce unnecessary expenses, it could be enough to propose one of these questionnaires (which also require a rather long time to be filled by parents, a fact which could lead to inaccuracies [26]).

IPDA questionnaire is highly economical, but our data show a correlation of its score with parameters which are not directly related to the child, such as the SocioEconomic Status of the family. Therefore, even if our study doesn't demonstrate any causal relation, it should be used cautiously.

The evaluation of the Socio-Economic Status, using a tool such as Hollingshead's Four Factor Index, is both cost-effective and interesting, given the correlations with many aspects of cognitive functioning and behaviour (evidenced both in scientific literature and in our data).

The Modified Bell Cancellation Test seems both lowcost and useful, but it is possible that its role is overestimated because the large majority of children enrolled had a poor attentive performance.
To end with, BVN 5-11 proved to be rather expensive, but is the only test included in our research protocol which allows us to obtain a sufficiently complete neuropsychological profile of the child, which is fundamental for planning a correct treatment strategy. It could be discussed, however, if this is the neuropsychological battery of choice or if others should be preferred.

\section{Conclusions}

A major and often claimed problem of the Italian Health System is the lack of resources. It is therefore important to choose evaluation tools on the basis of their ability to help understand children and their functional and diagnostic profile (efficacy) but also on their cost-effectiveness (efficiency).

Our study seems to offer valuable information not only on a non-clinical population of pre-schoolers, but also on the possibility of choosing a cost-effective evaluation protocol.

It must be said however that, although these tests are widely used in clinical practice, there is room for many improvements in terms of their cost-effectiveness but also of their psychometric properties, which are in general poorly defined. This represents a major problem not only for research but also, and most importantly, for clinical practice.

The major limitation of our study is that our data do not allow us to draw any conclusion on these psychometric properties, and therefore on the efficacy, of the tests used. This also limits our possibility to deepen the economical analysis, because a basic factor (i.e. test efficacy) is not completely defined.

\section{Acknowledgements}

The authors want to thank all teachers of the "Terzo Circolo Didattico" of Pavia and the Headmistress prof. Del Frate for their cooperation with this research project.

\section{Author details}

'Don Carlo Gnocchi ONLUS Foundation, Milan, Italy. ${ }^{2}$ Child Neuropsychiatry Unit, IRCCS "C. Mondino" Foundation, University of Pavia, Pavia, Italy. 


\section{Authors' contributions}

MC, EM, FP and UB planned the study. MC, EM, FP, ND and MT conducted children's evaluation. MC and UB conducted statistical analysis. All authors contributed to draft the manuscript, which they read and approved in the final version.

\section{Competing interests}

The authors declare that they have no competing interests.

Received: 28 March 2011 Accepted: 26 January 2012

Published: 26 January 2012

\section{References}

1. Tymms P: Baseline assessment and monitoring in Primary Schools: Achievements, Attitudes and Value-added Indicators London, David Fulton; 1999.

2. Tressoldi PE, Vio C: Il trattamento dei disturbi dell'apprendimento scolastico Trento, Erickson; 2002.

3. Hatcher PJ, Hulme C, Snowling MJ: Explicit phoneme training combined with phonic reading instruction helps young children at risk of reading failure. J Child Psychol Psych 2004, 45:338-358.

4. Geary DC, Hamson CO, Hoard MK: Numerical and arithmetical cognition: A longitudinal study of process and concept deficits in children with learning disability. J Exp Child Psychol 2000, 77(3):236-263.

5. Geary DC, Hoard MK, Hamson CO: Numerical and arithmetical cognition: Patterns of functions and deficits in children at risk for a mathematical disability. J Exp Child Psychol 1999, 74:213-239.

6. Termine C, Stella G, Capsoni C, Rosso E, Binda A, Pirola A, Conti C, Gruppi E, Lanzi G, Salini S, Tognatti C, Zoppello M, Balottin U: Neuropsychological profile of pre-schoolers with metaphonological difficulties: results from a non-clinical sample. Child Care Health Dev 2006, 33(6):703-12.

7. Gathercole SE, Pickering SJ, Knight C, Stegman Z: Working memory skills and educational attainment: Evidence from National Curriculum assessments at 7 and 14 years of age. App/ Cogn Psychol 2004, 18:1-16.

8. Grattan LM, Eslinger PJ: Long-term psychological consequences of childhood frontal lobe lesion in patient DT. Brain Cogn 1992, 20(1):185-195.

9. Blair C, Razza RP: Relating effortful control, executive function, and false belief understanding to emerging math and literacy ability in kindergarten. Child Dev 2007, 76:554-567.

10. Clark CA, Pritchard VE, Woodward LJ: Preschool executive functioning abilities predict early mathematics achievement. Dev Psychol 2010, 46(5):1176-1191

11. Chiappedi M, Balottin U, Baschenis IMC, Piazza F, Bernardi ED, Bejor M: Scientifically based nurture and nature: Alternative but non exclusive hypotheses on attention development. Med Hypotheses 2010, 75(5):445-447.

12. Shaywitz SE, Shaywitz BA: Paying attention to reading: The neurobiology of reading and dyslexia. Dev Psychopathol 2008, 20:1329-1349.

13. Cornoldi C: I disturbi dell'apprendimento Bologna, II Mulino; 1991.

14. Chiappedi M, Zoppello M, Rossi R, Scarabello EM, Piazza F: Specific learning disabilities and psychopathological aspects: the importance of early diagnosis. Minerva Pediatr 2007, 59(3):281-287.

15. Achenbach TM: Manual for the Child Behavior Checklist/2-3 and 1992 Profile Burlington, University of Vermont (Department of Psychiatry); 1992.

16. Nobile M, Alberti B, Zuddas A: Conners' Rating Scales - Revised (Adattamento italiano) Firenze, Giunti OS; 2007.

17. Hollingshed AB: Four factor index of social status. Yale Journal of Sociology 2011, 8:21-52

18. Corcella PR, Tretti ML, Terreni A, Tressoldi PE, Cornoldi C: IPDA - Questionario Osservativo per I' Identificazione Precoce delle Difficoltà di Apprendimento Trento, Erickson; 2002.

19. Belacchi C, Scalisi TG, Cannoni E, Cornoldi C: Manuale CPM. Coloured Progressive Matrices. Standardizzazione italiana Firenze, Giunti OS; 2008.

20. Stoppa E, Biancardi A: II test delle Campanelle Modificato: una proposta per lo studio dell'attenzione in età evolutiva. Psich Inf Adolesc 1997, 64:73-84.

21. Tressoldi PE, Vio C, Gugliotta M, Bisiacchi PS, Cendron M: BVN 5-11 - Batteria per la Valutazione Neuropsicologica per l'età evolutiva Trento, Erickson; 2005.

22. Mason L: Psicologia dell'apprendimento e dell'istruzione Bologna, II Mulino; 2006.
23. García-Molina A, Tirapu-Ustárroz J, Luna-Lario P, Ibáñez J, Duque P: Are intelligence and executive functions the same thing? Rev Neurol 2010, 50(12):738-746.

24. Hackman DA, Farah MJ, Meaney MJ: Socioeconomic status and the brain: mechanistic insights from human and animal research. Nat Rev Neurosci 2010, 11(9):651-659.

25. Althoff RR, Copeland WE, Stanger C, Derks EM, Todd RD, Neuman RJ, Van Beijsterveldt TC, Boomsma DI, Hudziak JJ: The latent class structure of ADHD is stable across informants. Twin Res Hum Genet 2006, 9(4):507-522.

26. Chiappedi M: Manuale di Psicometria Applicata Milano, Edizioni Anfora; 2009.

doi:10.1186/1824-7288-38-3

Cite this article as: Chiappedi et al: Abilities of preschoolers: comparing different tools. Italian Journal of Pediatrics 2012 38:3.

\section{Submit your next manuscript to BioMed Central and take full advantage of:}

- Convenient online submission

- Thorough peer review

- No space constraints or color figure charges

- Immediate publication on acceptance

- Inclusion in PubMed, CAS, Scopus and Google Scholar

- Research which is freely available for redistribution

Submit your manuscript at www.biomedcentral.com/submit 